\title{
Modeling Microwave Emission from Short Vegetation-Covered Surfaces
}

\section{Yanhui Xie ${ }^{1,2}$, Jiancheng Shi ${ }^{1,3, *}$, Yonghui Lei ${ }^{1,3, *}$ and Yunqing Li ${ }^{1,2}$}

1 State Key Laboratory of Remote Sensing Science, Institute of Remote Sensing and Digital Earth, Chinese Academy of Sciences, Beijing 100101, China; E-Mails: xieyh@ radi.ac.cn (Y.X.); liyunqing0317@163.com (Y.L.)

2 University of Chinese Academy of Sciences, Beijing 100049, China

3 Joint Center for Global Change Studies (JCGCS), Beijing 100875, China

* Authors to whom correspondence should be addressed; E-Mails: shijc@ @radi.ac.cn (J.S.); yonghui.lei@ hotmail.com (Y.L.); Tel.: +86-10-6480-7983 (J.S. \& Y.L.);

Fax: +86-10-6480-7983 (J.S. \& Y.L.).

Academic Editors: Alfredo R. Huete and Prasad S. Thenkabail

Received: 17 August 2015 / Accepted: 12 October 2015 / Published: 26 October 2015

\begin{abstract}
Owing to the temporal and spatial variability of the emissivity spectra, problems remain in the interpretation and application of satellite passive microwave data over vegetation-covered surfaces. The commonly used microwave land emissivity model, developed by Weng et al. (2001) and implemented into the community radiative transfer model (CRTM), treats vegetation-covered surfaces as a three-layer medium. This simplification comes at the cost of accuracy. In this study, to reduce bias in the modeling of microwave emissions from short vegetation-covered surfaces, two modifications are made. First, vegetation was considered as a multilayered medium including leaves and stems to simulate volumetric absorption and scattering. The results suggest that the calculated brightness temperatures well agree with field experiments under different incidence angles for low soil moisture and sparse crop cover. On the other hand, large errors from the measurements are found for high soil moisture content and dense crop cover. Second, the advanced integral equation model (AIEM) was also used to improve the simulation of reflectivity from rough soil surfaces. Comparisons with field experimental data show that the determination coefficient between the calculated and measured brightness temperatures significantly increased and the root-mean-square errors
\end{abstract}


remarkably decreased. The average improvement using the proposed approach is about $80 \%$ and $59 \%$ in accuracy for the vertical and horizontal polarization, respectively.

Keywords: microwave emissivity; short vegetation-covered surfaces; multilayered medium; two-stream radiative transfer approximation

\section{Introduction}

Passive microwave radiometers can provide valuable information for monitoring the global temperature, moisture, biomass, and structure of vegetation canopies. Satellite microwave brightness temperature observations over oceans and the atmosphere have been widely used to improve the numerical weather predictions. However, problems remain in the interpretation and application of satellite passive microwave data over different land surfaces owing to the temporal and spatial variability of the emissivity spectra [1].

Microwave radiation of a scene depends on the thermodynamic temperature of the emitting medium and its emissivity [2]. Thus, satellite microwave signals are sensitive to the surface emissivity. A surface emissivity uncertainty of 5\%-10\% will produce a brightness temperature uncertainty up to several degrees [3]. Microwave emission signals from vegetation-covered surfaces are affected by many factors. Vegetation-covered surfaces are complex media, whose microwave emission includes contributions from the soil and vegetation layers. The emission characteristics of vegetation are directly linked to the integrated water content of the layer and the dimensions and structure of the vegetation components [4,5]. Vegetation comprises leaves, stems, branches, and even trunks, which are often represented as canonical shapes like disks and cylinders. A number of studies discussed the features of optical parameters for different vegetation shapes [6-8]. For instance, they studied leaves, trigs, branches, and even trunks, as disk- and needle-shaped and cylindrical. Ground-based and airborne radiometric measurements over low frequencies have been conducted to improve our understanding of the interaction between the microwave signals and vegetation [9-13]. The effect of soil cannot be neglected. The emission of bare soil is a function of the surface physical conditions, such as soil moisture content, soil temperature, soil texture, and soil surface roughness, which also depend on the observation frequency [14].

Modeling techniques based on radiative transfer theory have been developed to interpret the characteristics of microwave emission from vegetation-covered surfaces. Simple approaches treat vegetation as a uniform layer based on the single-scattering albedo $[9,15,16]$. One of the most known models is the L-band microwave emission of the biosphere (L-MEB) model, which was the result of an extensive review of the current knowledge of microwave emissions of various land covers [17]. In the models, the dimension and shape of vegetation scatterers are assumed equal; therefore, the canopy can be characterized by two global parameters: the single-scattering albedo $\omega$ and the optical thickness $\tau[4,18]$. On the other hand, sophisticated radiative transfer schemes are proposed to account for multiple scattering effects $[4,19]$. These schemes estimate the attenuation characteristics including volume absorption and scattering from the discrete scatterers of the vegetation elements. The contributions of the different scatterers are then combined by means of the double matrix 
method [4,20]. Representing vegetation as a single continuum element is only valid under certain conditions, whereas an ensemble of discrete scattering elements can provide a more correct representation of vegetation $[4,15]$. For rough surfaces, such as the lower boundary condition of the vegetation layer, physical modeling and parameterized approaches are commonly used to simulate the surface reflectivity [21]. The applicability of parameterized surface reflectivity models has limitations because they are often calibrated on limited data. For this reason, it may be preferable to physically model the rough surface reflectivity [21,22]. According to the physical parameters from vegetation and boundary conditions at the soil surface, the theoretical microwave emissivity from canopy can be obtained by solving the equation of radiative transfer.

The accuracy of modeling emissivity depends on the availability of highly accurate input data ranging from soil moisture to property of vegetation elements. However, the fundamental problem is that accurate input data are not available on larger scales. Nevertheless, previous studies have shown that it is critical to correctly present the effects of vegetation properties and soil surface roughness in modeling microwave emissivity spectra from vegetation-covered surfaces. This study aims to improve the accuracy of modeling microwave emission from short vegetation-covered surfaces based on the commonly used microwave land emissivity model developed by Weng (referred to as the Weng model) [1]. Modification is offered to determine the microwave emissivity over vegetation-covered surfaces. It focuses on improving the modeling of two aspects of microwave emission, the attenuation characteristics of vegetation as an ensemble of different scattering elements and the reflectivity of rough soil surfaces. The attenuation characteristics of vegetation are derived by considering vegetation as a multilayered medium, including stems and leaves. The simulation of rough surface reflectivity is improved using the advanced integral equation model (AIEM) [23]. The brightness temperatures calculated by modeling the microwave emission are validated by measurements over short vegetationcovered surfaces, mainly including soybean and cotton fields.

\section{Methodology}

In the Weng model, a vegetation-covered surface is represented as a three-layer medium. The volumetric scattering of vegetation is calculated using two-stream radiative transfer. Geometric optics is applied to compute the optical parameters for canopy [24], which only considers the effects from leaves. The rough surface reflection is derived by a parameterized model modifying the Fresnel reflection coefficients [14].

The three-layer medium Weng model is shown in Figure 1 [1]. The top layer 1 stands for air with uniform permittivity $\varepsilon_{1}$. The bottom layer 3 is the subsurface with permittivity $\varepsilon_{3}$. Vegetation belongs to the middle layer 2 with spatially homogeneous scatterers and bulk permittivity $\varepsilon_{2}$. $\tau$ is the optical thickness, $\mu$ is the cosine of the incident zenith angle, and $R_{i j}$ is the reflectivity at the interfaces of different layers. $I_{0}$ is the downwelling radiance at $\tau=\tau_{0}$ from the top layer. $I_{1}$ is the upwelling radiance at $\tau=\tau_{1}$ from the bottom layer. $B$ is the Planck function and $T$ is the thermal temperature. 


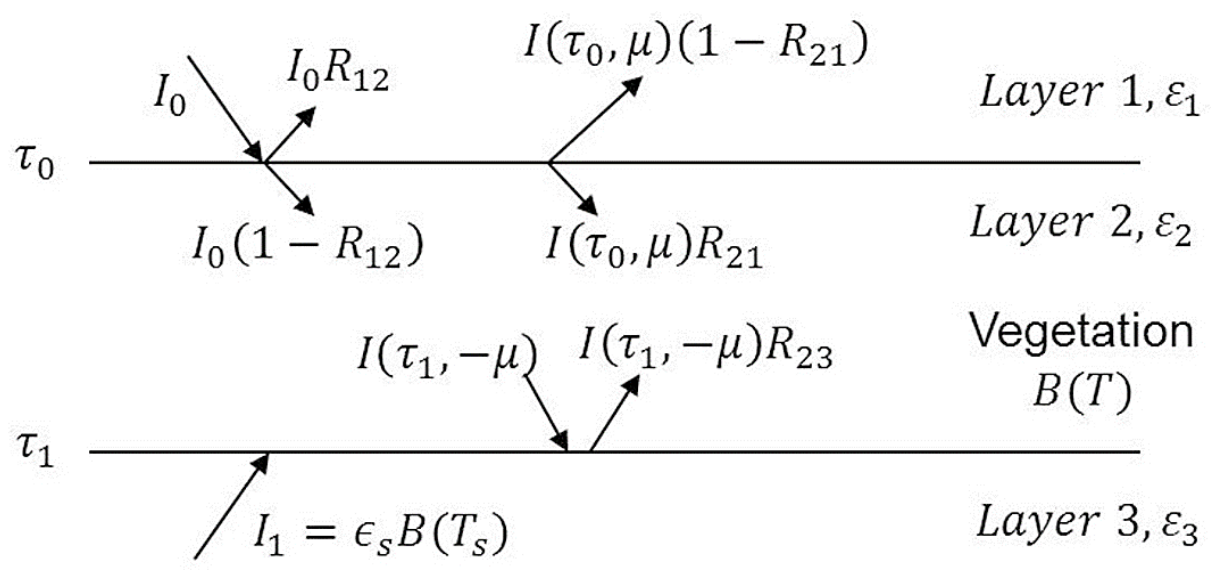

Figure 1. Schematic of the radiative transfer for the three-layer medium.

Based on the volumetric scattering of vegetation, the surface reflectivity, the three-layer medium, and the two-stream radiative transfer approximation, the emissivity for vegetation-covered surfaces can be described as [1].

$$
\epsilon=\alpha R_{12}+\left(1-R_{21}\right) \frac{(1-\beta)\left[1+\gamma e^{-2 \kappa\left(\tau_{1}-\tau_{0}\right)}\right]+\alpha\left(1-R_{12}\right)\left[\beta-\gamma e^{-2 \kappa\left(\tau_{1}-\tau_{0}\right)}\right]}{\left(1-\beta R_{21}\right)-\left(\beta-R_{21}\right) \gamma e^{-2 \kappa\left(\tau_{1}-\tau_{0}\right)}}
$$

where $\alpha=I_{0} / B, \beta=(1-a) /(1+a), a=\sqrt{(1-\omega)(1-\omega g)}$, and $\gamma=\left(\beta-R_{23}\right) /\left(1-\beta R_{23}\right) \cdot g$ is the asymmetry factor for a scattering particle, $\omega$ is the single-scattering albedo, and $\kappa$ is the eigenvalue of the two-stream solution and $\kappa=a / \mu$.

The microwave land emissivity model developed by Weng has been coupled with the community radiative transfer model (CRTM) to simulate the radiance of a scene [1]. However, the microwave radiances over land simulated with CRTM are rarely used in assimilation and soil moisture retrieval owing to the uncertainty of radiance from vegetation-covered surfaces and the higher computational demands.

Based on the Weng model, modification is proposed to improve the accuracy of modeling the emissivity spectra from short vegetation-covered surfaces. First, the proposed approach considers vegetation as a mixture of discrete elements (stems) and a continuous medium (leaves) overlaying a rough surface. Finite cylinders are used to model the stems of short vegetation. Leaves are represented by the leaf area index ( $L A I)$ and thickness. Their effects are then combined to obtain the optic parameters from the entire vegetation layer. Second, physical modeling is used to obtain the reflectivity from a rough surface rather than the parameterized method in the Weng model. Finally, the above obtained effects of vegetation and soil are input into Equation (1) to improve the modeling of the emissivity spectra from short vegetation-covered surfaces.

\subsection{Vegetation Volume Scattering}

Different methods have been developed to model the electromagnetic properties of single scatterers for vegetation components. Different shapes, such as disks and cylinders, are generally assumed to represent dielectric elements of vegetation single scatterers. The Rayleigh-Gans approximation is used to estimate the disks cross sections at low frequencies [25]. At high frequencies, the internal field of dielectric disks is calculated using the physical optics approximation [26]. Geometric optics is used for canopy leaves to compute the optical parameters in a wide frequency range (1-100 GHz) [24], and the 
results were much improved in comparison with the commonly used Rayleigh approximation [18]. The scattering and absorption from stems are derived using the infinite length cylinder approximation [27]. Their contributions are then considered together in the emissivity model.

\subsubsection{The Geometric Optics Approach for Leaves}

In the geometric optics approach, the canopy leaves are approximated as a homogeneous slab with uniform permittivity $\varepsilon_{v e g}$ and thickness $d(\mathrm{~mm})$. For a single leaf, the reflectivity, transmissivity, and absorptivity $R_{p}, T_{p}$, and $A_{p}$, respectively, where $p$ is $v$ or $h$ and stands for the vertical or horizontal polarization, respectively, are given as follows $[1,24]$ :

$$
\begin{gathered}
R_{p}=\left|\frac{r_{p}\left(1-e^{-i 2 k_{z 1} d}\right)}{1-r_{p}^{2} e^{-i 2 k_{z 1} d}}\right|^{2} \\
T_{h}=\left|\frac{4 k_{z 0} k_{z 1} e^{i\left(k_{z 0}-k_{z 1}\right) d}}{\left(k_{z 0}+k_{z 1}\right)^{2}\left(1-r_{h}^{2} e^{-i 2 k_{z 1} d}\right)}\right|^{2} \\
T_{v}=\left|\frac{4 \varepsilon_{v e g} k_{z 0} k_{z 1} e^{i\left(k_{z 0}-k_{z 1}\right) d}}{\left(\varepsilon_{v e g} k_{z 0}+k_{z 1}\right)^{2}\left(1-r_{v}^{2} e^{-i 2 k_{z 1} d}\right)}\right|^{2} \\
A_{p}=1-R_{p}-T_{p}
\end{gathered}
$$

where $i=\sqrt{-1}$ and

$k_{0}=2 \pi / \lambda$,

$k_{z 0}=k_{0} \cos \beta$,

$k_{z 1}=k_{0}\left(\varepsilon_{v e g}-\sin ^{2} \beta\right)^{1 / 2}$,

$r_{h}=\left(k_{z 0}-k_{z 1}\right) /\left(k_{z 0}+k_{z 1}\right)$,

$r_{v}=\left(\varepsilon_{v e g} k_{z 0}-k_{z 1}\right) /\left(\varepsilon_{v e g} k_{z 0}+k_{z 1}\right)$.

$k$ stands for the wavenumber $\left(\mathrm{mm}^{-1}\right), \lambda$ is the free-space wavelength $(\mathrm{mm}), \beta$ is the incidence angle $\left({ }^{\circ}\right)$ relative to the leaf normal. Permittivity $\varepsilon_{v e g}$ is obtained using the mixing formula, which treats the leaf as a matrix of bound water, saline water, and dry matter [5].

\subsubsection{Infinite Cylinder Approximation for Stems}

The vegetation stems are considered as finite-length dielectric cylinders. The scattering amplitude tensor for a finite-length cylinder is derived by estimating the corresponding field inside a similar cylinder of infinite length. The cylinder's principal axes are aligned along a local frame of axes $\hat{x}$, $\hat{y}$, and $\hat{z}$ in the infinite-length cylinder approximation. The local frame can be oriented with respect to the fixed frame of coordinates, which is the reference frame of the entire canopy. Given the radius $a(\mathrm{~m})$, length $2 h(\mathrm{~m})$, and relative permittivity $\varepsilon_{r}$ for the dielectric cylinder, the scattering amplitude tensor $f_{p q}(\hat{S}, \hat{\imath})$ and absorption cross-section $Q_{a p}$ in the local frame of the cylinder are written as [27,28] 


$$
\begin{aligned}
& f_{p q}(\hat{s}, \hat{\imath})=2 k^{2} h \mu(\hat{s}, \hat{\imath})\left(\varepsilon_{r}\right. \\
& -1) \sum_{n=-\infty}^{\infty} e^{j n\left(\emptyset_{s}-\emptyset_{i}\right)}\left\{\frac { k } { 2 \lambda _ { i } } \left[\left(\eta h_{n q}-j e_{n q} \cos \theta_{i}\right)\left(\hat{p}_{s} \cdot \hat{x}+\hat{p}_{s} \cdot \hat{y}\right) z_{n+1} e^{j \emptyset_{s}}-\left(\eta h_{n q}\right.\right.\right. \\
& \left.\left.\left.+j e_{n q} \cos \theta_{i}\right)\left(\hat{p}_{s} \cdot \hat{x}-\hat{p}_{s} \cdot \hat{y}\right) z_{n-1} e^{-j \emptyset_{s}}\right]+e_{n q} z_{n}\left(\hat{p}_{s} \cdot \hat{z}\right)\right\} \\
& Q_{a p}=\operatorname{scale} \cdot 4 \pi k h \varepsilon_{r} \sum_{0}^{\infty}\left(\left|e_{n p}\right|^{2} y_{n}+2\left|c_{n p}\right|^{2} y_{n+1}+2\left|d_{n p}\right|^{2} y_{n-1}\right)
\end{aligned}
$$

where $\hat{\imath}\left(\theta_{i}, \emptyset_{i}\right)$ is the incident direction, $\hat{s}\left(\theta_{s}, \emptyset_{s}\right)$ is the scattering direction, $p$ and $q$ stand for the polarizations, and $\hat{p}_{s}$ is the polarization vector. $j=\sqrt{-1}$,

$$
\begin{aligned}
& \mu(\hat{s}, \hat{\imath})=\frac{\sin \left[k h\left(\cos \theta_{i}+\cos \theta_{s}\right)\right]}{k h\left(\cos \theta_{i}+\cos \theta_{s}\right)}, \\
& z_{n}=\frac{a^{2}}{u^{2}-v_{s}{ }^{2}}\left[u J_{n}\left(v_{s}\right) J_{n+1}(u)-v_{s} J_{n}(u) J_{n+1}\left(v_{s}\right)\right], \\
& u=\lambda_{i} a=k a \sqrt{\varepsilon_{r}-\cos ^{2} \theta_{i}}, \\
& v_{i}=k a \sin \theta_{i}, \\
& v_{s}=k a \sin \theta_{s}, \\
& e_{n v}=\frac{j \sin \theta_{i}}{R_{n} J_{n}(u)}\left\{\frac{H^{\prime}{ }_{n}{ }^{(2)}\left(v_{i}\right)}{v_{i} H_{n}{ }^{(2)}\left(v_{i}\right)}-\frac{J^{\prime}{ }_{n}(u)}{u J_{n}(u)}\right\}, \\
& e_{n h}=\frac{-\sin \theta_{i}}{R_{n} J_{n}(u)}\left(\frac{1}{v_{i}{ }^{2}}-\frac{1}{u^{2}}\right) n \cos \theta_{i}, \\
& \eta h_{n v}=\frac{\sin \theta_{i}}{R_{n} J_{n}(u)}\left(\frac{1}{v_{i}{ }^{2}}-\frac{1}{u^{2}}\right) n \cos \theta_{i}, \\
& \eta h_{n h}=\frac{j \sin \theta_{i}}{R_{n} J_{n}(u)}\left\{\frac{H^{\prime}{ }_{n}{ }^{(2)}\left(v_{i}\right)}{v_{i} H_{n}{ }^{(2)}\left(v_{i}\right)}-\frac{\varepsilon_{r} J^{\prime}{ }_{n}(u)}{u J_{n}(u)}\right\}, \\
& R_{n}=\frac{\pi v_{i}{ }^{2} H_{n}{ }^{(2)}\left(v_{i}\right)}{2}\left\{\left(\frac{H^{\prime}{ }_{n}{ }^{(2)}\left(v_{i}\right)}{v_{i} H_{n}{ }^{(2)}\left(v_{i}\right)}-\frac{J^{\prime}{ }_{n}(u)}{u J_{n}(u)}\right) \cdot\left(\frac{H^{\prime}{ }_{n}{ }^{(2)}\left(v_{i}\right)}{v_{i} H_{n}{ }^{(2)}\left(v_{i}\right)}-\frac{\varepsilon_{r} J^{\prime}{ }_{n}(u)}{u J_{n}(u)}\right)-n^{2} \cos ^{2} \theta_{i}\left(\frac{1}{u^{2}}-\frac{1}{v_{i}{ }^{2}}\right)^{2}\right\},
\end{aligned}
$$

where $J_{n}$ is the cylindrical Bessel function and $H_{n}{ }^{(2)}$ is the Hankel function. If $n=0$, the scale $=1$; else $n>0$, scale $=2$ for $Q_{a p}$, and

$c_{n p}=\frac{k\left(\eta h_{n p}-j e_{n p} \cos \theta_{i}\right)}{2 \lambda_{i}}$,

$d_{n p}=\frac{k\left(\eta h_{n p}+j e_{n p} \cos \theta_{i}\right)}{2 \lambda_{i}}$,

$y_{n}=\frac{a}{\omega^{2}-\bar{\omega}^{2}}\left[\omega J_{n}(\bar{u}) J_{n+1}(u)-\bar{\omega} J_{n+1}(\bar{u}) J_{n}(u)\right]$,

$\omega=k \sqrt{\varepsilon_{r}-\cos ^{2} \theta_{i}}$,

$u=a \omega$.

The scattering amplitude tensor $F_{p q}$ in the reference frame is obtained through the Euler angles of rotation

$$
F_{p q}=T_{s} f_{p q} T_{i}
$$




$$
\begin{gathered}
T_{i}=\left[\begin{array}{cc}
-t_{v i} & -t_{h i} \\
t_{h i} & -t_{v i}
\end{array}\right] / \sqrt{t_{v i}{ }^{2}+t_{h i}{ }^{2}} \\
T_{s}=\left[\begin{array}{ll}
-t_{v s} & t_{h s} \\
-t_{h s} & -t_{v s}
\end{array}\right] / \sqrt{t_{v s}{ }^{2}+t_{h s}{ }^{2}}
\end{gathered}
$$

where $t_{v i}$ and $t_{h i}$ correspond to the functions of the incident and Euler angles, respectively and $t_{v s}$ and $t_{h s}$ correspond to the functions of the scattering and Euler angles, respectively. Finally, the scattering $\left(Q_{s p}{ }^{*}\right)$ and absorption $\left(Q_{a p}{ }^{*}\right)$ cross sections for the cylinder in the reference frame are

$$
\begin{gathered}
Q_{s p}{ }^{*}=\int_{4 \pi}\left(\left|F_{v p}\right|^{2}+\left|F_{h p}\right|^{2}\right) d \Omega \\
Q_{a p}{ }^{*}=\left(Q_{a p} t_{p i}{ }^{2}+Q_{a q} t_{q i}{ }^{2}\right) /\left(t_{p i}{ }^{2}+t_{q i}{ }^{2}\right)
\end{gathered}
$$

\subsubsection{Vegetation Optic Parameters}

The contributions from leaves and stems are considered together in the radiative transfer. Thus, the scattering $\left(k_{s p}\right)$ and absorption $\left(k_{a p}\right)$ coefficients for the entire vegetation layer are calculated as follows:

$$
\begin{aligned}
& k_{s p}=\frac{L A I}{H} \cdot \int_{0}^{\pi / 2} R_{p} \cos \xi \cdot n(\xi) d \xi+N \cdot Q_{s p}{ }^{*} \\
& k_{a p}=\frac{L A I}{H} \cdot \int_{0}^{\pi / 2} A_{p} \cos \xi \cdot n(\xi) d \xi+N \cdot Q_{a p}{ }^{*}
\end{aligned}
$$

where $L A I$ is the leaf area index $\left(\mathrm{m}^{2} \cdot \mathrm{m}^{-2}\right), H$ is the canopy depth $(\mathrm{m})$ including leaves and stems, $\xi$ is the leaf orientation angle, and $N$ represents the number of cylinders in unit volume. The single-scattering albedo $\omega_{p}$ and the optical thickness $\tau_{p}$ for vegetation are described as

$$
\begin{gathered}
\omega_{p}=k_{s p} /\left(k_{s p}+k_{a p}\right) \\
\tau_{p}=\left(k_{s p}+k_{a p}\right) \cdot H
\end{gathered}
$$

\subsection{Rough Surface Reflection}

Surface reflection is an essential component affecting the emissivity from vegetation-covered surfaces. The reflectivity of soil surface forms important boundary conditions for the reflected vegetation emission [29,30]. Physical modeling and semiempirical approaches are commonly used to model the surface reflectivity or emission. The method used in the Weng model is a parameterized scheme based on limited field observations [14]. Compared with parameterized empirical schemes and other early theoretical models, the AIEM model has the ability to produce accurate simulations of the surface emission signals over a wider range of surface roughness, soil moisture, and incidence angles [21]. Therefore, the AIEM model is used to calculate the rough surface reflectivity in this study.

In the AIEM model, the scattering fields from rough surfaces include three terms [23]: Kirchhoff fields, complementary fields, and a cross term of them. The surface effective reflectivity is expressed as

$$
R_{p}{ }^{e}=\frac{1}{4 \pi \cos \theta_{i}} \int_{0}^{2 \pi} \int_{0}^{\pi / 2}\left[\sigma_{p p}^{s}\left(\theta_{i}, \phi_{i} ; \theta_{s}, \phi_{s}\right)+\sigma_{q p}^{s}\left(\theta_{i}, \phi_{i} ; \theta_{s}, \phi_{s}\right)\right] \sin \theta_{s} d \theta_{s} d \phi_{s}
$$


where $\sigma^{S}$ is the bistatic scattering coefficient, subscript $p$ or $q$ describes the polarization state, $\left(\theta_{i}, \emptyset_{i}\right)$ is the incident direction, and $\left(\theta_{s}, \emptyset_{s}\right)$ is the scattering direction.

\section{Field Experimental Data}

Field experimental data were collected by the State Key Laboratory of Remote Sensing Science, Beijing Normal University. Experiments were conducted at the crop field test site of Qingyuan (Hebei) in the North of China at $38.7^{\circ} \mathrm{N}, 115.4^{\circ} \mathrm{E}$. A truck-mounted multifrequency microwave radiometer (TMMR) by Radiometer Physics GmbH (Germany) was used in all measurements. The TMMR comprises antennas, the positioner, the host software, and the platform [31,32], as shown in Figure 2 (left). Microwave brightness temperatures from crop fields were measured in parallel and perpendicular to the truck carrying the radiometer. The TMMR was operated in four channels at $6.925,10.65,18.7$, and $36.5 \mathrm{GHz}$ (also named band $\mathrm{C}, \mathrm{X}, \mathrm{Ku}$, and $\mathrm{Ka}$, respectively) at vertical $(V)$ and horizontal $(H)$ polarizations. The beam width with $-3 \mathrm{~dB}$ antenna gain for radiometer height of $4.95 \mathrm{~m}$ was between $13.7^{\circ}$ at $6.925 \mathrm{GHz}$ and $20.5^{\circ}$ at $36.5 \mathrm{GHz}$, as in Figure 2 (right). The absolute instrument calibration was conducted by the four-point calibration procedure before the experiments [32]. Accuracy of $\pm 1 \mathrm{~K}$ and a sensitivity of about $\pm 0.5 \mathrm{~K}$ was achieved with the aid of calibration target temperature sensors and the minimization of thermal gradients [31,33].
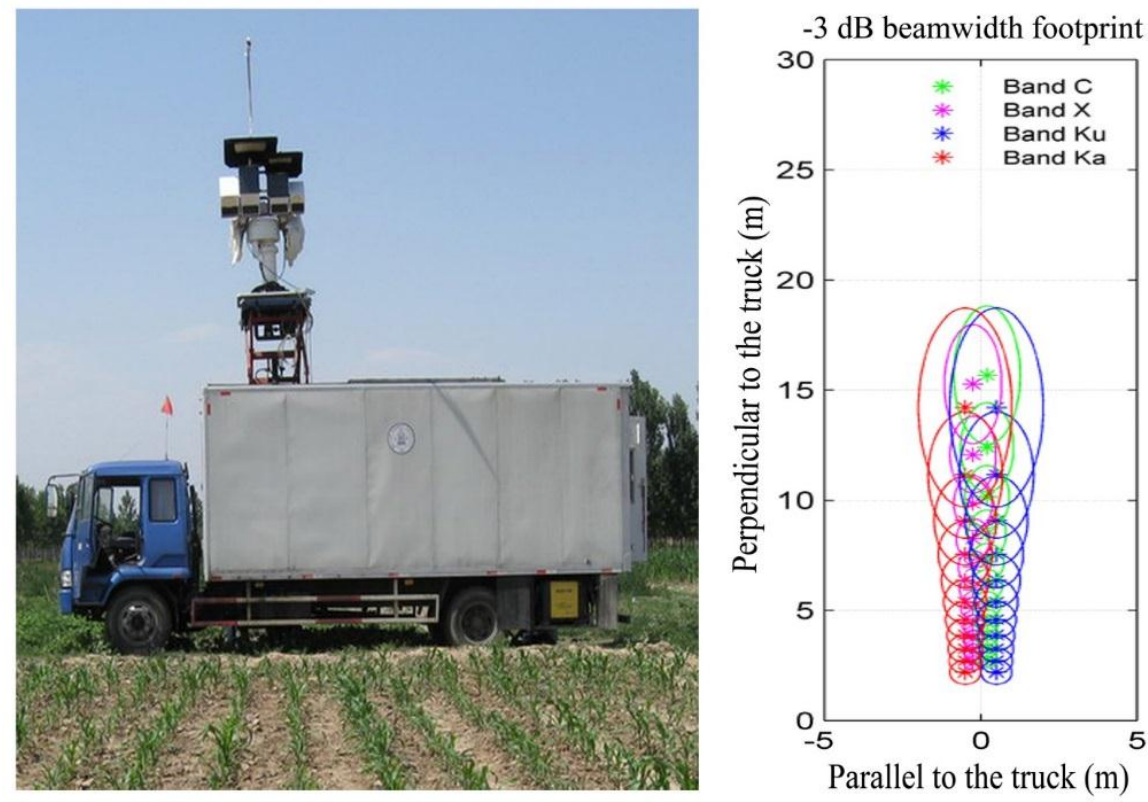

Figure 2. A truck-mounted multifrequency microwave radiometer (TMMR) radiometer system (left) and the footprint with $-3 \mathrm{~dB}$ beamwidth (right).

The TMMR radiometer was set to work at incident angles between $20^{\circ}$ and $70^{\circ}$ at $5^{\circ}$ increments and used to obtain the microwave brightness temperatures of crop fields. The vegetation and soil moisture content were measured by the traditional weighting method. There was no significant change in soil moisture during the observations; thus, the average value of $0-1 \mathrm{~cm}$ was used as the effective value [32]. LAI was calculated by the number and size of leaves and stems, which were manually measured. A platinum resistance thermometer was used to measure the temperature of vegetation and 
soil, and the mean value of $0-5 \mathrm{~cm}$ below the surface was taken as the effective soil temperature. The surface roughness was confirmed by using a grid plate and a digital camera.

The experiments were conducted on different dates in the summer of 2009; soybean on 23 June and 9 July, and cotton on 10 June and 23 June, as shown in Figure 3. All measured parameters are listed in Table 1.

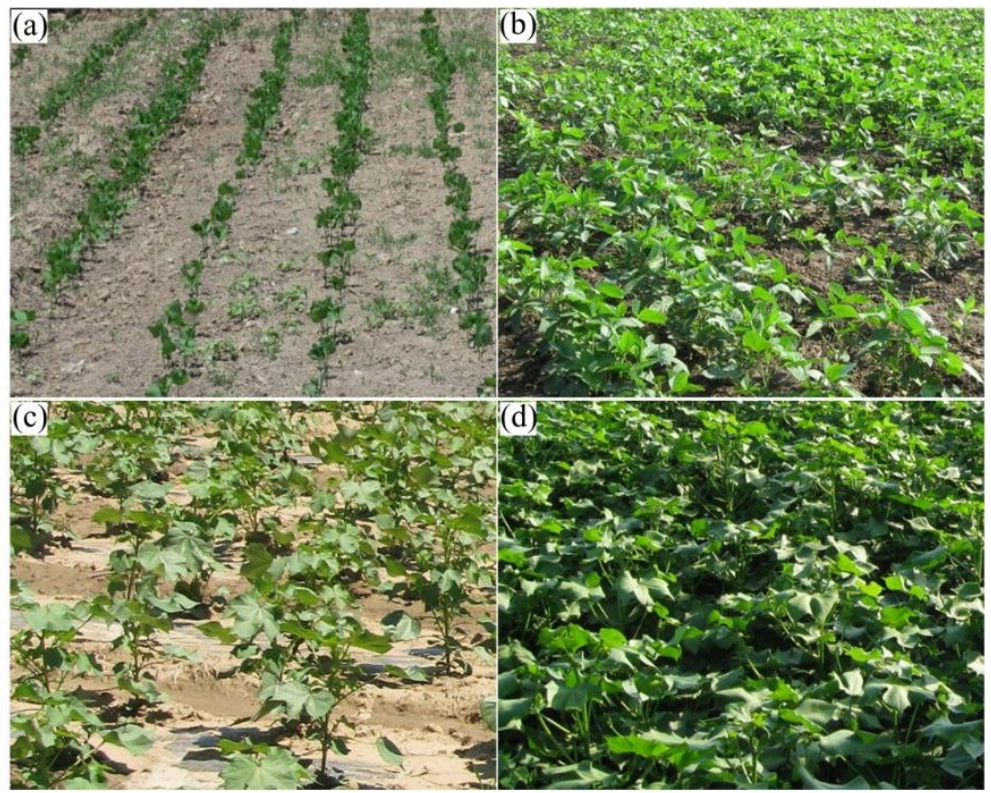

Figure 3. Soybean and cotton fields on different dates used in the TMMR measurements. (a) Soybean on 23 June; (b) soybean on 9 July; (c) cotton on 10 June; and (d) cotton on 23 June.

Table 1. Parameters for different vegetation parts and rough soil surface in the experiments (LAI: leaf area index; $M g$ : gravimetric moisture; $S M C$ : soil moisture content; and $R M S$ : standard deviation of surface height).

\begin{tabular}{cccccc}
\hline \multirow{2}{*}{ Scatterers } & \multirow{2}{*}{ Measured Parameters } & \multicolumn{2}{c}{ Soybean } & \multicolumn{2}{c}{ Cotton } \\
\cline { 3 - 6 } & & 23 June & 9 July & 10 June & 23 June \\
\hline Vegetation & Depth $(\mathrm{m})$ & 0.11 & 0.33 & 0.19 & 0.37 \\
& Temperature $\left({ }^{\circ} \mathrm{C}\right)$ & 36.8 & 29.4 & 26.3 & 29.4 \\
\hline \multirow{2}{*}{ Leaves } & $L A I\left(\mathrm{~m}^{2} \cdot \mathrm{m}^{-2}\right)$ & 0.58 & 1.35 & 0.71 & 1.57 \\
& Thickness $(\mathrm{mm})$ & 0.31 & 0.38 & 0.23 & 0.27 \\
& $M g\left(\mathrm{~g} \cdot \mathrm{g}^{-1}\right)$ & 0.85 & 0.75 & 0.82 & 0.80 \\
\hline Stems & Radius $(\mathrm{m})$ & 0.0009 & 0.0013 & 0.0026 & 0.003 \\
& Length $(\mathrm{m})$ & 0.05 & 0.08 & 0.08 & 0.15 \\
& $M g\left(\mathrm{~g} \cdot \mathrm{g}^{-1}\right)$ & 0.88 & 0.82 & 0.88 & 0.90 \\
& Density $\left(\mathrm{m}^{-2}\right)$ & 277 & 378 & 285 & 327 \\
& Angle distribution & oblique & oblique & oblique & oblique \\
\hline Rough soil & $S M C\left(\mathrm{~cm}^{3} \cdot \mathrm{cm}^{-3}\right)$ & 0.0138 & 0.162 & 0.30 & 0.05 \\
surface & $R M S$ height $(\mathrm{m})$ & 0.03 & 0.03 & 0.02 & 0.03 \\
& Correlation length $(\mathrm{m})$ & 0.09 & 0.09 & 0.1 & 0.1 \\
& Skin temperature $\left({ }^{\circ} \mathrm{C}\right)$ & 49.5 & 33.2 & 34.5 & 42.1 \\
& Soil temperature $\left({ }^{\circ} \mathrm{C}\right)$ & 43.1 & 32.9 & 31.5 & 33.6 \\
\hline
\end{tabular}




\section{Results and Discussion}

\subsection{Parameters Sensitivity}

First, the sensitivity of vegetation optic parameters in the proposed modeling approach is analyzed. Figure 4 shows the polarization characteristics of the single-scattering albedo $\omega_{p}$ and optical depth $\tau_{p}$ for vegetation according to the group of vegetation features in Table 2. Bands $\mathrm{C}, \mathrm{X}$, and $\mathrm{Ku}$ correspond to frequencies of $6.925 \mathrm{GHz}, 10.7 \mathrm{GHz}$, and $18.7 \mathrm{GHz}$, respectively, which are also used by the TMMR system. The results presented in Figure 4 suggest that the single-scattering albedo shows distinct polarization characteristics for leaf thickness $d$ between $0.1 \mathrm{~mm}$ and $0.4 \mathrm{~mm}$. With increasing leaf thickness, it increases for both $V$ and $H$ polarizations. The single-scattering albedo of $H$ is higher than the corresponding $V$ at $\omega_{v}$ below 0.6 except for $d=0.1 \mathrm{~mm}$, where both are close to the $1: 1$ line. The difference increases with the leaf thickness and decreases with increasing $\omega_{v}$. When leaves are thin with thickness $0.1 \mathrm{~mm}$, the single-scattering albedo of $H$ is lower than that of $V$ at $\omega_{v}$ above 0.5 . With increasing frequency, $\omega_{v}$ shifts toward low values and $\omega_{h}$ increases slightly. Optical depth $\tau_{h}$ is systematically higher than $\tau_{v}$. The difference increases with optical depth especially for the $\mathrm{X}$ and $\mathrm{Ku}$ bands. The range of optical depth decreases for $V$ and $H$ with increasing frequency.

Table 2. Parameters of vegetation features used in the polarization analysis (LAI: leaf area index and $M g$ : gravimetric moisture).

\begin{tabular}{cccccc}
\hline Scatterers & Parameters & Minimum & Maximum & Interval & Number \\
\hline Leaves & $L A I\left(\mathrm{~m}^{2} \cdot \mathrm{m}^{-2}\right)$ & 0.5 & 5.5 & 1.0 & 6 \\
& Thickness $(\mathrm{mm})$ & 0.1 & 0.4 & 0.1 & 4 \\
& $M g\left(\mathrm{~g} \mathrm{~g}^{-1}\right)$ & 0.60 & 0.80 & 0.10 & 3 \\
\multirow{5}{*}{ Stems } & Radius $(\mathrm{m})$ & 0.002 & 0.022 & 0.004 & 6 \\
& Height (m) & 0.2 & 1.0 & 0.2 & 5 \\
& $M g\left(\mathrm{~g}^{-1}\right)$ & 0.50 & 0.70 & 0.10 & 3 \\
& Density $\left(\mathrm{m}^{-2}\right)$ & 50 & 200 & 50 & 4 \\
\hline
\end{tabular}
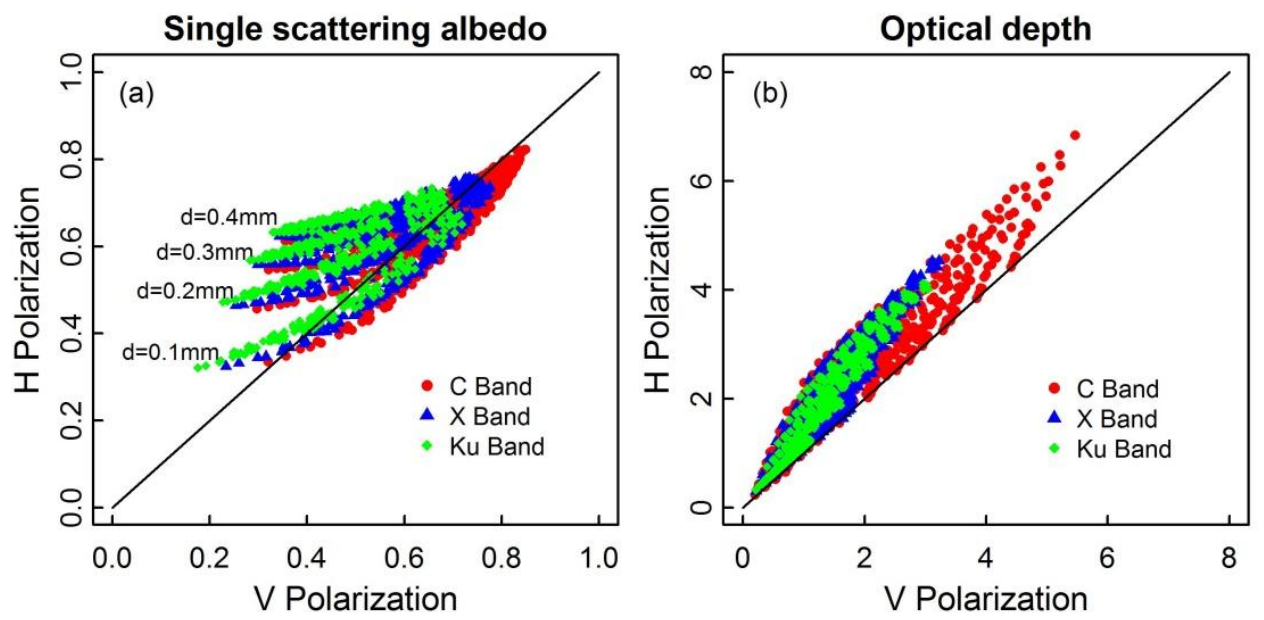

Figure 4. Polarization characteristics of single-scattering albedo and optical depth for the vegetation in the improved model. (a) Single-scattering albedo and (b) optical depth. 
The emissivity estimated with the proposed modeling approach is analyzed versus incidence angle and frequency for different soil moisture content $\left(S M C, \mathrm{~cm}^{3} \cdot \mathrm{cm}^{-3}\right)$, with parameters $a=0.005 \mathrm{~m}, h=0.2 \mathrm{~m}, L A I=1.56 \mathrm{~m}^{2} \cdot \mathrm{m}^{-2}, d=0.27 \mathrm{~mm}$, and $M g=0.80 \mathrm{~g} \cdot \mathrm{g}^{-1}$. Figure 5 shows that the emissivity slightly increases for $V$ polarization and decreases for $H$ polarization with increasing incidence angle. It becomes also clear that the polarization differences increase with the incidence angles. With increasing frequency, the emissivity for $V$ and $H$ polarizations shows the same trend as the dry soil at high incidence angles. Moreover, for $V$ polarization with high soil moisture, the incidence angle has a stronger effect compared with that for the dry soil scenario. In Figure 6, the emissivity sharply decreases as frequency increases up to $10 \mathrm{GHz}$ and varies slightly above $10 \mathrm{GHz}$. High soil moisture content causes the emissivity to decrease, especially for a low incidence angle of $20^{\circ}$. This is consistent with the results in Figure 5.
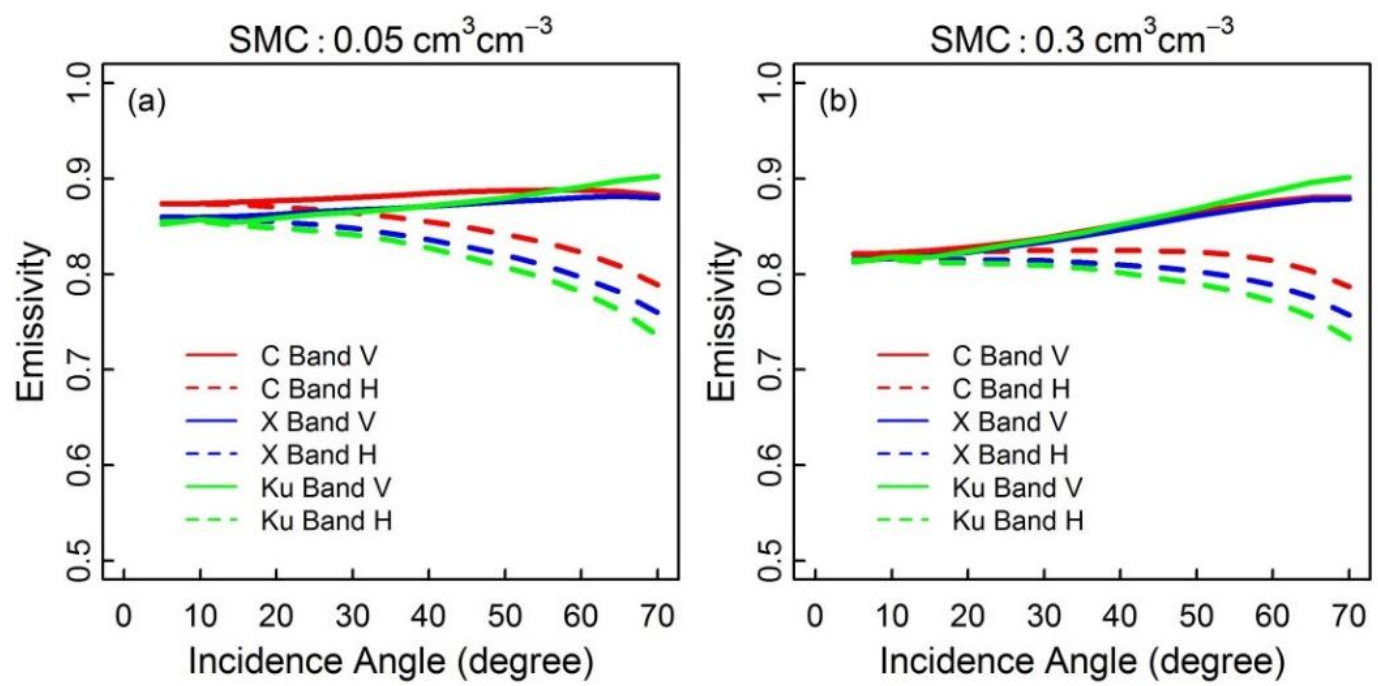

Figure 5. Estimated emissivity versus incidence angle for different soil moisture contents $(S M C)$ (a) $S M C: 0.05 \mathrm{~cm}^{3} \cdot \mathrm{cm}^{-3}$ and (b) $S M C: 0.30 \mathrm{~cm}^{3} \cdot \mathrm{cm}^{-3}$.
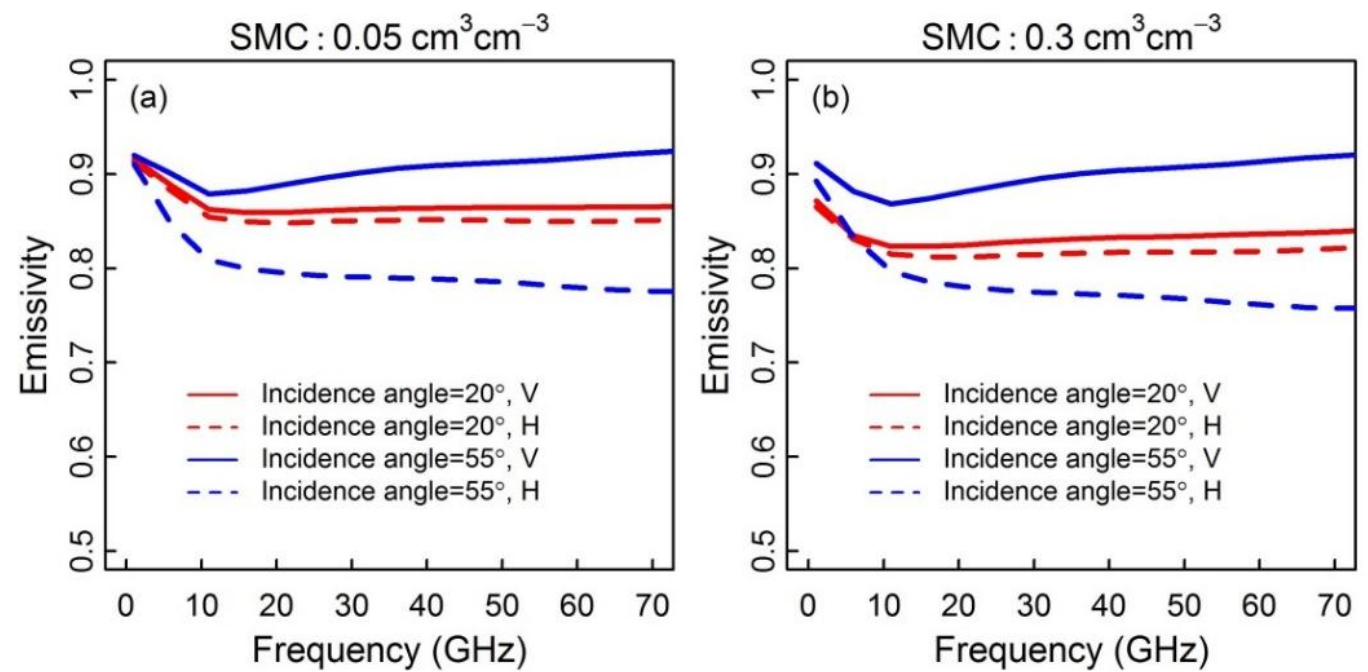

Figure 6. Estimated emissivity versus frequency for different soil moisture content (SMC) for (a) $S M C: 0.05 \mathrm{~cm}^{3} \cdot \mathrm{cm}^{-3}$ and (b) $S M C: 0.30 \mathrm{~cm}^{3} \cdot \mathrm{cm}^{-3}$. 


\subsection{Comparisons with Field Experimental Data}

In this section, brightness temperatures from short vegetation-covered surfaces calculated by the improved microwave emissivity model are compared with the field experimental results of the TMMR. In general, the brightness temperature of short vegetation-covered surface is the product of emissivity and physical temperature [34,35]. For physical temperature, the mean value of soil and vegetation temperatures was used.
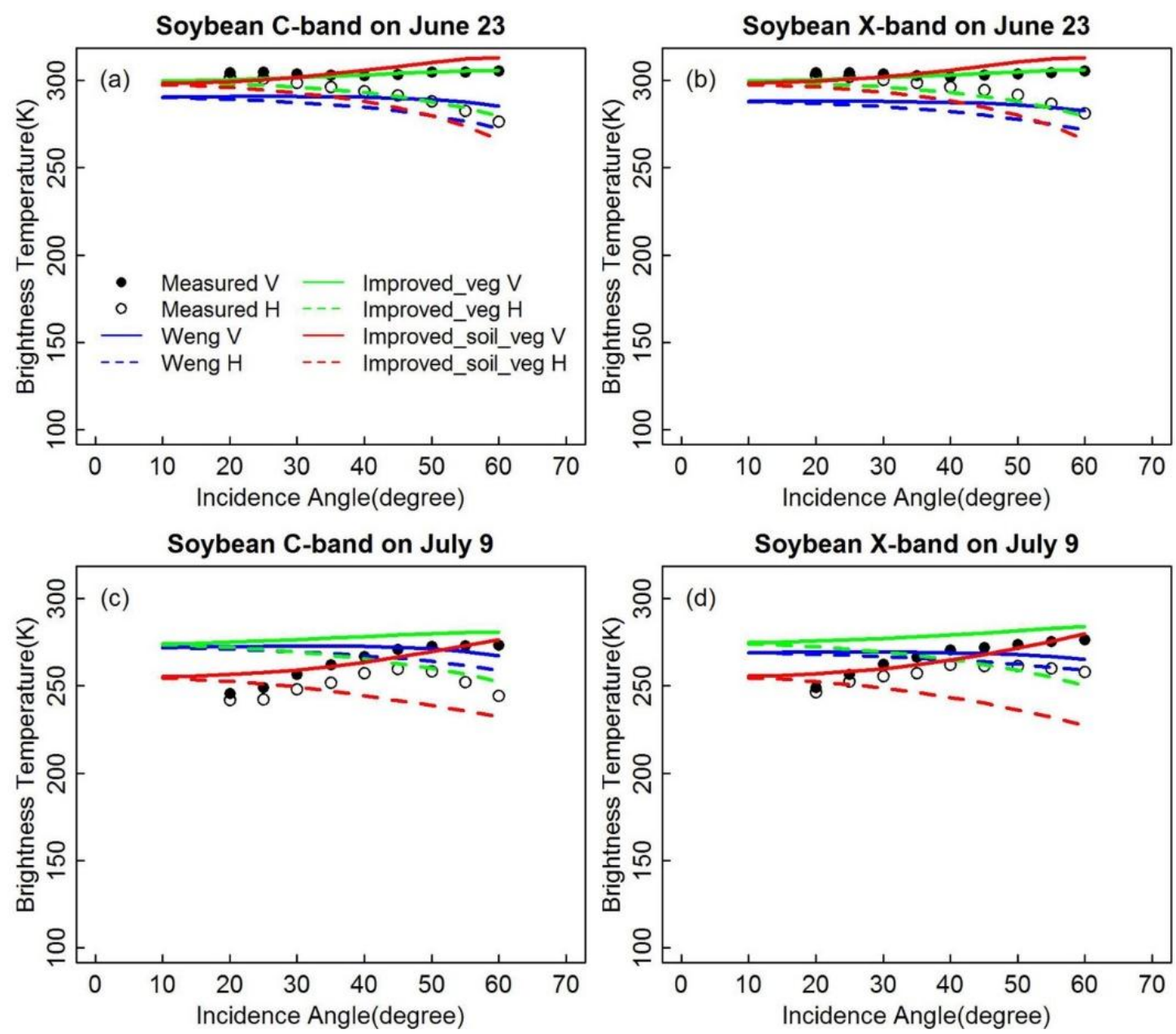

Figure 7. Comparisons between the measured brightness temperature and the brightness temperature for the model for soybean at different frequencies and on different dates.

(a) Soybean for C band on 23 June 2009; (b) Soybean for X band on 23 June 2009;

(c) Soybean for C band on 9 July 2009; and (d) Soybean for X band on 9 July 2009.

Comparisons between the measured brightness temperature and the brightness temperature for the model for soybean are given in Figure 7. Weng indicates the initial Weng emissivity model in Figure 7, improved_veg stands for the model wherein the radiative transfer only improved for vegetation, and improved_soil_veg is the improved model including improvements in vegetation and the surface based on Weng model, respectively. Compared with the measurements, brightness temperatures calculated with the Weng model are generally lower for both $\mathrm{C}$ and $\mathrm{X}$ bands on 23 June whereby the difference is slightly larger for the $\mathrm{X}$ band. In contrast, improved_veg and improved_soil_veg well agree with the measurements for soybean on 23 June except at high incidence angles. On 9 July the results from the 
Weng and improved_veg models show large deviations from the measurement at low incidence angles. In contrast, the results for the $V$ polarization from the improved_soil_veg model show better agreement with the measurements for both the $\mathrm{C}$ and $\mathrm{X}$ bands but slightly larger difference for $H$ polarization at high incidence angles on 9 July.

Cotton C-band on June 10

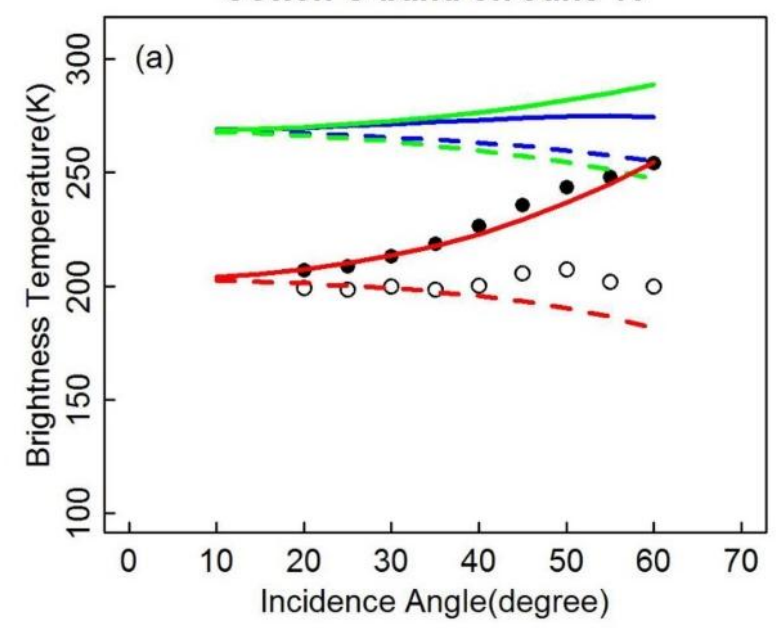

Cotton C-band on June 23

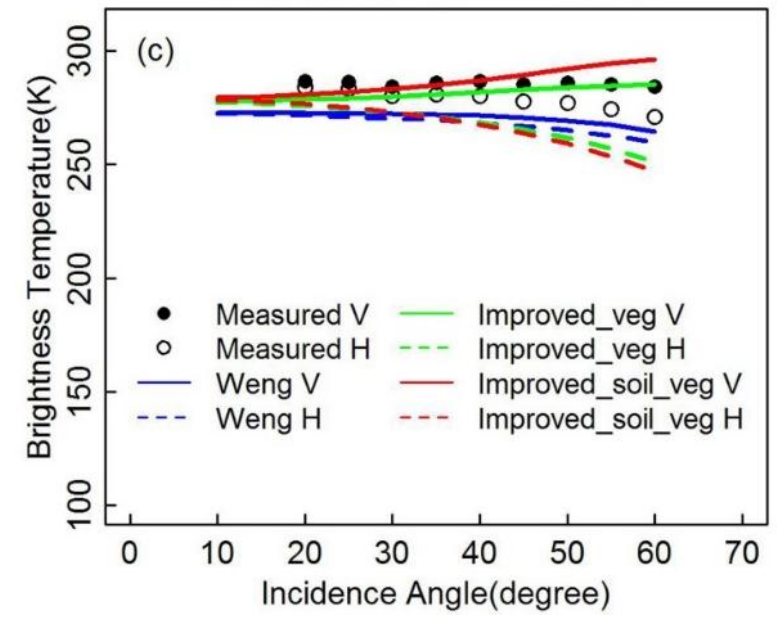

Cotton X-band on June 10

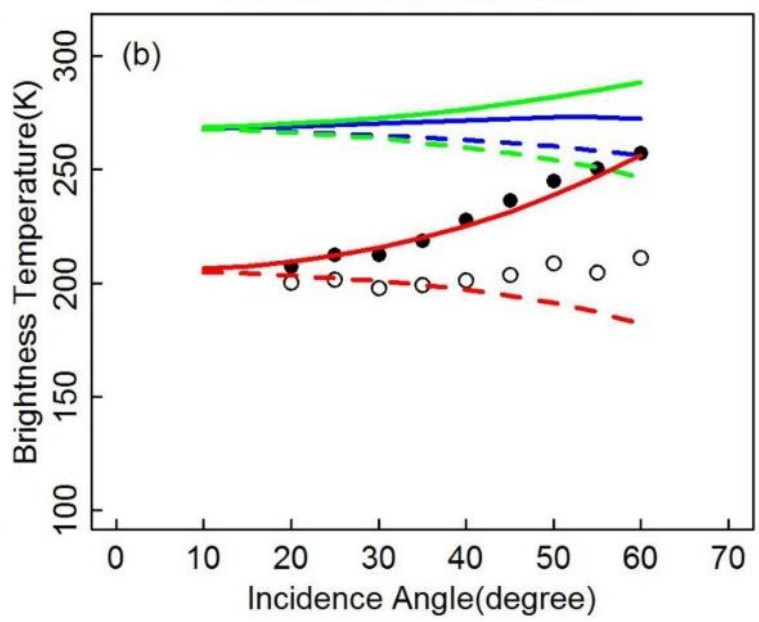

Cotton X-band on June 23

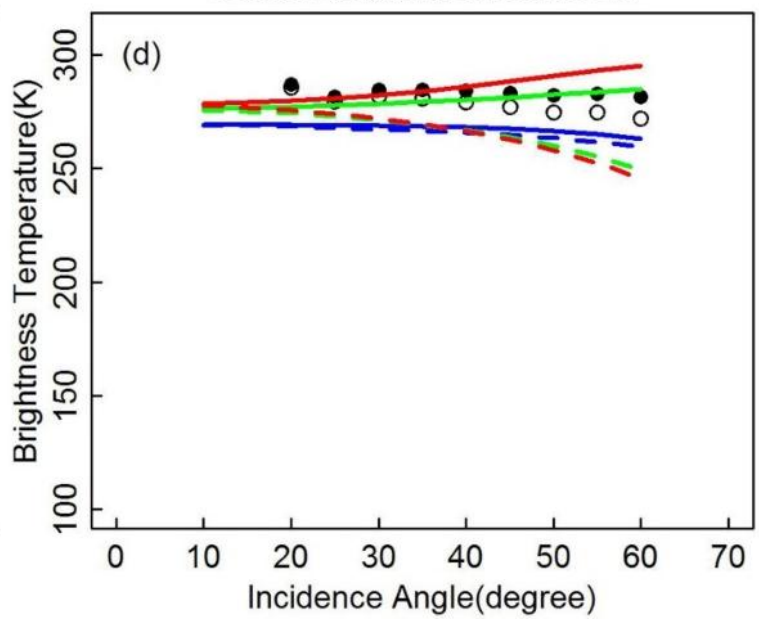

Figure 8. Comparisons between measured brightness temperature and the brightness temperature for the model for cotton at different frequencies and on different dates. (a) Cotton for C band on 10 June 2009; (b) Cotton for X band on 10 June 2009; (c) Cotton for C band on 23 June 2009; and (d) Cotton for X band on 23 June 2009.

Figure 8 shows the results for the cotton field on two different dates. A distinct difference between the brightness temperature of the model and the measured brightness temperature is seen for both $V$ and $H$ polarizations for the Weng and improved_veg models on 10 June 2009. Similar differences are detected for the $\mathrm{C}$ and $\mathrm{X}$ bands. The improved_soil_veg model on the other hand significantly improved the model results, only small differences for $H$ polarization are seen at high incidence angles $>40^{\circ}$. For the measurement on 23 June, the measured brightness temperatures are high for both $V$ and $H$ polarizations, whereas the Weng model generally underestimates the measured data. The improved_veg model agrees with the measurements for the $V$ polarization but not for $H$. With increasing incidence angle, the 
brightness temperatures of the improved_soil_veg model exhibit large differences between the two polarizations and high inconsistency with the measurements.

Table 3 lists the statistics for different models and the field experimental measurements at different frequencies and polarizations. The results suggest that the determination coefficient $\mathrm{R}^{2}$ of the improved_veg model increases for the $V$ polarization but shows little improvement for the $H$ polarization, compared with that of the Weng model. For the improved_soil_veg model, $\mathrm{R}^{2}$ is higher than 0.9 for both $V$ and $H$ polarizations for the $\mathrm{C}$ and $\mathrm{X}$ bands. For the $V$ polarization, $\mathrm{R}^{2}$ is slightly higher than that for the $H$ polarization. The root-mean-square errors (RMSEs) for the improved_veg and the Weng models are similar at $\geq 25 \mathrm{~K}$. Moreover, the RMSEs of the improved_soil_veg model also show significant improvement, especially for the $V$ polarization at $5.03 \mathrm{~K}$ for the $\mathrm{C}$ band and at $5.19 \mathrm{~K}$ for the $\mathrm{X}$ band, with improvements of $81.1 \%$ and $79.8 \%$, respectively. The RMSE values of the $H$ polarization for the improved_soil_veg model decreased to $11.73 \mathrm{~K}$ for the $\mathrm{C}$ band and $14.92 \mathrm{~K}$ for the $\mathrm{X}$ band, compared with those of $>30 \mathrm{~K}$ for the Weng model, with improvements of $64.3 \%$ and $53.0 \%$, respectively. The average improvement in accuracy from the improved_soil_veg model is about $80 \%$ for the $V$ polarization and $59 \%$ for the $H$ polarization.

Table 3. Statistics for the brightness temperature (K) with the Weng, improved_veg, and improved_soil_veg models and field experimental data at different frequencies and polarizations.

\begin{tabular}{cccccccc}
\hline & \multirow{2}{*}{ Models } & \multicolumn{2}{c}{ Weng Model } & \multicolumn{2}{c}{ Improved_veg } & \multicolumn{2}{c}{ Improved_soil_veg } \\
\cline { 3 - 8 } Statistics & & C Band & X Band & C Band & X Band & C Band & X Band \\
\hline \multirow{2}{*}{$\mathrm{R}^{2}$} & $\mathrm{~V}$ & 0.33 & 0.27 & 0.62 & 0.60 & 0.98 & 0.97 \\
& $\mathrm{H}$ & 0.50 & 0.42 & 0.49 & 0.44 & 0.95 & 0.93 \\
\multirow{2}{*}{$\mathrm{RMSE}$} & $\mathrm{V}$ & 26.67 & 25.70 & 26.71 & 25.71 & 5.03 & 5.19 \\
& $\mathrm{H}$ & 32.81 & 31.77 & 30.86 & 29.67 & 11.73 & 14.92 \\
\hline
\end{tabular}

\subsection{Discussion}

\subsubsection{General Discussion of Results}

The microwave radiative transfer over vegetation-covered surfaces is a complex process and includes multiple components. The absorption and scattering properties of the elements are critical for modeling the microwave radiation signals from vegetation-covered surfaces. Ferrazzoli et al. $[4,36]$ pointed out that the soil is the dominant emission source at low frequencies, whereas the upper elements dominate at high frequencies. In this study, the attenuation characteristics of vegetation, including leaves and stems, showed sensitivity to the frequency and polarization direction. The single-scattering albedo is dominated by the leaf thickness, whereas the effect of stems is weak but not negligible. The optical depth of vegetation varies with frequency and presents a small dynamical range at high frequencies. The total emissivity also depends on frequency. It decreases rapidly as frequency increases up to $10 \mathrm{GHz}$, which should be due to a dominant scattering effect of the vegetation layer. The results also suggest that the modeling of microwave emissivity is particularly sensitive to the incidence angle and soil moisture. 

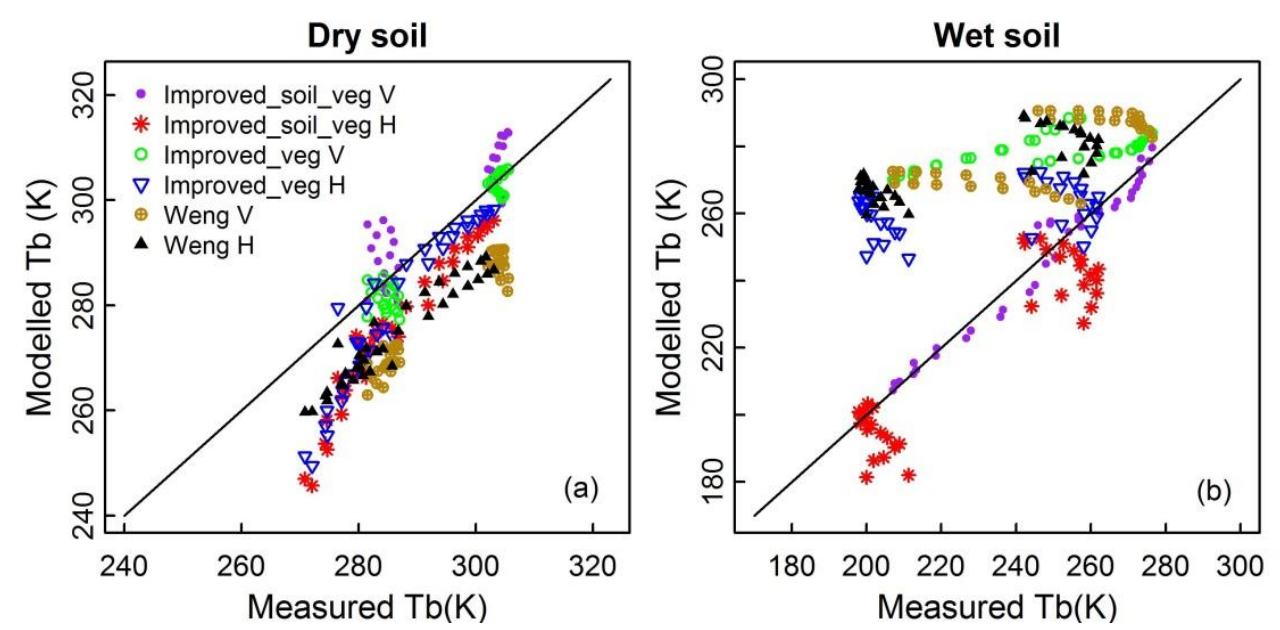

Figure 9. Brightness temperature of model versus measured brightness temperature $(\mathrm{Tb})$ for (a) dry soil: including crop fields for soybean with $S M C 0.0138 \mathrm{~cm}^{3} \mathrm{~cm}^{-3}$ and cotton with $S M C 0.05 \mathrm{~cm}^{3} \cdot \mathrm{cm}^{-3}$ on 23 June; (b) wet soil: including crop fields soybean with $S M C$ $0.162 \mathrm{~cm}^{3} \cdot \mathrm{cm}^{-3}$ on 9 July and cotton with $S M C 0.3 \mathrm{~cm}^{3} \cdot \mathrm{cm}^{-3}$ on 10 June.

Table 4. Statistics for the Weng, improved_veg, and improved_soil_veg models' brightness temperature versus measured brightness temperature $(\mathrm{K})$ under dry and wet soil conditions.

\begin{tabular}{cccccccc}
\hline & \multirow{2}{*}{ Models } & \multicolumn{2}{c}{ Weng Model } & \multicolumn{2}{c}{ Improved_veg } & \multicolumn{2}{c}{ Improved_soil_veg } \\
\cline { 3 - 8 } Statistics & & Dry Soil & Wet Soil & Dry Soil & Wet Soil & Dry Soil & Wet Soil \\
\hline \multirow{2}{*}{$\mathrm{R}^{2}$} & $\mathrm{~V}$ & 0.94 & 0.06 & 0.94 & 0.46 & 0.75 & 0.97 \\
& $\mathrm{H}$ & 0.92 & 0.08 & 0.90 & 0.08 & 0.93 & 0.83 \\
\multirow{2}{*}{$\mathrm{RMSE}$} & $\mathrm{V}$ & 16.06 & 33.37 & 3.63 & 36.90 & 5.91 & 4.15 \\
& $\mathrm{H}$ & 12.28 & 43.99 & 9.85 & 41.66 & 12.32 & 14.44 \\
\hline
\end{tabular}

The validation and comparison of the different microwave emissivity modeling approaches for short vegetation-covered surfaces suggest that only improvements in the radiative transfer offer agreement with the experimental measurements at low soil moisture (dry soil), as shown in Figure 9a. The RMSEs (in Table 4) of the improved_veg model decreased significantly for dry soil compared with those of the Weng model, especially for the $V$ polarization. Large errors are detectable at high soil moisture (wet soil), as in Figure 9b; moreover, the radiation in the $H$ polarization is not well represented by the improved_veg model for dense crop cover (cotton on 23 June). The improvements in vegetation and soil provide much better agreement between model and measured data over a wider range of conditions. In particular, there is remarkable improvement for high soil moisture (Table 4) and dense crops. The data analysis highlights several points. First, the effects from stems cannot be neglected when modeling the microwave emission from short vegetation-covered surfaces. Considering only the effect of vegetation leaves, as in the Weng model, introduces relatively large errors. Second, the rough surface reflectivity calculated by the AIEM model better represents its dependences on soil moisture, incidence angle, and frequency over a wide range of conditions. This is consistent with previous studies [21-23]. The Weng model, wherein a parameterized surface reflectivity method is used, shows large error for high soil moisture and low incidence angles. 
Compared with the Weng model, the improved model significantly reduces the errors. In addition, owing to the low values of $L A I$ taken into consideration, the emissivity from crop covers is dominated by the soil contribution. This is well evident in the case of wet soil. Moreover, multiple scattering effects increase for dense vegetation and consequently affect the accuracy of microwave emission modeling.

\subsubsection{Sources of Uncertainty}

In microwave emission modeling of short vegetation-covered surfaces based on the Weng model, two improvements in vegetation and soil are considered. Comparisons with field experimental measurements suggest an average improvement of about $80 \%$ and 59\% in accuracy, respectively, for $V$ and $H$ polarizations. However, the errors are still relatively large at high incidence angles, especially for the $H$ polarization. The uncertainties and errors of the results mainly depend on the accuracy of the experimental measurements and the modeling method.

First, measurement errors are inevitable. The measurements of the input parameters, such as leaf thickness, cylinder radius and stem length, surface RMS height, and correlation length, are performed manually in the field experiments. Owing to the complexity of vegetation, the characteristic distribution of leaves and stems is not uniform. Therefore, representative sample selection is difficult, if not impossible. In our experiments, the input parameters for all scatterers are averaged values of selected samples; that is, the effective soil temperature is averaged from the vertical temperature profiles over $0-5 \mathrm{~cm}$ depth, and the mean value from the soil and vegetation temperatures is used as the physical temperature. These average values were used as the "true" values for soil and vegetation cover. In addition, systematic errors from instruments and the calibration methods can also affect the accuracy of the experimental data. All the above add to the uncertainties of the comparisons between calculated and measured brightness temperatures.

Second, natural soil and vegetation are strongly heterogeneous spatially. Homogenous dielectric properties and isotropic surface roughness are assumed in the microwave emission modeling of short vegetation-covered surfaces. The assumption also adds to the uncertainty.

In addition, only single scattering of leaves and stems is considered for the vegetation in the modeling. This is valid for sparse vegetation, whereas it does not hold for dense vegetation where the scatterers are close to each other and multiple scattering takes place, especially at high frequencies [4,37]. Multiple scattering is not considered in this study, which may be a source of uncertainty in the case of dense vegetation.

Apart from the above uncertainties, two limitations hinder the applicability of the modeling approach. First, the model is considerably complex despite the significant improvement in accuracy in the modeling of the microwave emission from short vegetation-covered surfaces. A simpler parameterization method may be a good compromise. Second, it is difficult to obtain the specified input parameters owing to different vegetation types and their properties at different growth stages. One feasible approach would be to obtain the input parameters through a number of training vegetation samples according to different types and growth stages. Therefore, further modeling efforts should be focused on the simplification of the model algorithm, training the input parameters samples for different vegetation types, and their validations. However, a fundamental problem in the application of 
the proposed emissivity model should highlight the need for highly accurate input data ranging from soil moisture to leaf thickness and orientation, which will never be available at large scales.

\section{Conclusions}

This study aims to improve the accuracy of modeling the microwave emission from vegetation-covered surfaces based on the Weng model. A modeling approach that considered both vegetation elements and rough soil surfaces was developed. Contributions from leaves and stems were used to derive the attenuation characteristics of vegetation in the radiative transfer. The AIEM model was used to improve the simulation of reflectivity from rough soil surfaces, and the microwave emissivity from vegetation-covered surfaces was obtained through a two-stream radiative transfer approximation solution. The improvements were validated for short vegetation-covered surfaces against field experimental measurements, and model uncertainties were analyzed.

The results indicate that microwave emissivity modeling of vegetation-covered surfaces depends on vegetation elements, soil moisture, incidence angle, and frequency. The validation of the modeling approach for short vegetation-covered surfaces showed good agreement with experimental data with the correlation coefficient greater than 0.9. The comparisons with the Weng model suggest that the improvements in the modeling of vegetation and soil significantly increased the determination coefficient between the calculated and measured brightness temperatures and decreased the RMSEs. However, the applicability of the modeling approach might be limited owing to its uncertainties and complexity, including the unavailability of highly accurate input data ranging from soil moisture to leaf thickness and orientation.

Further studies are necessary to validate the advantage and limitations of the modeling approach. In the current study, the verification of the improved emissivity model is based on two types of crops (soybean and cotton) at different times. More observational measurements for other vegetation types are needed in the future.

\section{Acknowledgments}

This study is sponsored by Strategic Priority Research Program for Space Sciences of the Chinese Academy of Sciences (XDA04061200), National Key Basic Research Program of China (2015CB953701), the CAS/SAFEA international partnership program for creative research teams (KZZD-EW-TZ-09) and the earth system process spatial information simulation and extreme climatic event characteristic study (Y3SG1700CX).

\section{Author Contributions}

Yanhui Xie wrote the paper; Jiancheng Shi supervised the study and reviewed the manuscript; Yonghui Lei supervised, reviewed and edited the manuscript; Yunqing Li provided the related data and part of the code. All authors read and approved the manuscript.

\section{Conflicts of Interest}

The authors declare no conflict of interest. 


\section{References}

1. Weng, F.Z.; Yan, B.H.; Grody, N.C. A microwave land emissivity model. J. Geophys. Res. 2001, 106, 20115-20123.

2. Paloscia, S. Microwave emission from vegetation. In Passive Microwave Remote Sensing of Land-Atmosphere Interactions; Choudhury, B.J., Kerr, Y.H., Njoku, E.G., Pampaloni, P., Eds.; VSP Press: Utrecht, The Netherlands, 1995; pp. 357-374.

3. Weng, F.Z.; Grody, N.C. Physical retrieval of land surface temperature using the special sensor microwave imager. J. Geophys. Res. 1998, 103, 8839-8848.

4. Ferrazzoli, P.; Guerriero, L. Modeling microwave emission from vegetation-covered surfaces: A parametric analysis. In Passive Microwave Remote Sensing of Land-Atmosphere Interactions; Choudhury, B.J., Kerr, Y.H., Njoku, E.G., Pampaloni, P., Eds.; VSP Press: Utrecht, The Netherlands, 1995; pp. 389-402.

5. Ulaby, F.T.; El-Rayes, M.A. Microwave dielectric spectrum of vegetation. Part II: Dual-dispersion model. IEEE Trans. Geos. Remot. Sens. 1987, GE-25, 550-556.

6. Karam, M.A.; Fung, A.K.; Lang, R.H.; Chuahan, N.S. A microwave scattering model for layered vegetation. IEEE Trans. Geosci. Remote Sens. 1992, 30, 767-784.

7. Ulaby, F.T.; Sarabandi; McDonald, K.; Whitt, M.; Dobson, M.C. Michigan canopy scattering model. Int. J. Remote Sens. 1990, 11, 1223-1253.

8. Tsang, L.; Kong, J.A.; Shin, R.T. Radiative transfer theory for active remote sensing ellipsoidal scatters. Radio Sci. 1984, 19, 629-642.

9. Ulaby, F.T.; Moore, R.K.; Fung, A.K. Passive microwave sensing of land. In Microwave Remote Sensing Active and Passive; Artech House Press: Dedham, MA, USA, 1986; Volume III, pp. 1522-1638.

10. Pampaloni, P.; Paloscia, S. Microwave emission and plant water content: A comparison between field measurements and theory. IEEE Trans. Geosci. Remote Sens. 1986, GE-24, 900-905.

11. Wegmuller, U. Remote Sensing Signature Studies on Agricultural Fields with Ground-based Radiometry and Scatterometry. Ph.D. Thesis, University of Berne, Bern, Switzerland, 1990.

12. Schwank, M.; Matzler, C.; Guglielmetti, M.; Fluhler, H. L-band radiometer measurements of soil water under growing clover grass. IEEE Trans. Geosci. Remote Sens. 2005, 43, 2225-2237.

13. Vereecken, H.; Weihermüller, L.; Jonard, F.; Montzka, C. Characterization of crop canopies and water stress related phenomena using microwave remote sensing methods: A review. Vadose Zone J. 2012, 11, doi:10.2136/vzj2011.0138ra.

14. Choudhury, B.J.; Schmugge, T.J.; Chang, A.; Newton, R.W. Effect of surface roughness on the microwave emission from soils. J. Geophys. Res. 1979, 84, 5699-5706.

15. Au, W.C.; Tsang, L.; Shin, R.T.; Kong, J.A. Collective scattering and absorption in microwave interaction with vegetation canopies. Prog. Electromagn. Res. 1996, 14, 181-231.

16. Kurum, M.; Lang, R.H.; O’Neill, P.E.; Joseph, A.T.; Jackson, T. A first-order radiative transfer model for microwave radiometry of forest canopies at L-band. IEEE Trans. Geosci. Remote Sens. 2011, 49, 3167-3179. 
17. Wigneron, J.P.; Kerr, Y.H.; Waldtufe, P.; Saleh, K.; Richaume, P. L-band microwave emission of the biosphere (L-MEB) model: Results from calibration against experimental data sets over crop fields. Remote Sens. Environ. 2007, 107, 639-655.

18. Mo, T.; Choudhury, B.J.; Schmugge, T.J.; Wang, J.R.; Jackson, T.J. A model for microwave emission from vegetation-covered fields. J. Geophys. Res. 1982, 87, 11229-11237.

19. Fung, A.K.; Eom, H.J. Emission from a rayleigh layer with irregular boundaries. J. Quant. Spectrosc. Radiat. Trans. 1981, 26, 397-409.

20. Ferrazzoli, P.; Solimini, D.; Luzi, G.; Paloscia, S. Model analysis of backscatter and emission from vegetated terrains. JEWA 1991, 5, 175-193.

21. Shi, J.C.; Jiang, L.M.; Zhang, L.X.; Chen, K.S.; Wigneron, J.P.; Chanzy, A. A parameterized multifrequency-polarization surface emission model. IEEE Trans. Geosci. Remote Sens. 2005, 43, 2831-2841.

22. Zhao, T.J.; Shi, J.C.; Bindlish, R.; Jackson, T.; Cosh, M.; Jiang, L.M. Parametric Exponentially Correlated Surface Emission Model For L-Band Passive Microwave Soil Moisture Retrieval. Available online: http://dx.doi.org/10.1016/j.pce.2015.04.001 (accessed on 15 May 2015).

23. Chen, K.S.; Wu, T.D.; Tsang, L.; Li, Q.; Shi, J.C.; Fung, A.K. The emission of rough surfaces calculated by the integral equation method with a comparison to a three-dimensional moment method simulations. IEEE Trans. Geosci. Remote Sens. 2003, 41, 90-101.

24. Wegmuller, U.; Matzler, C.; Njoku, E. Canopy opacity models. In Passive Microwave Remote Sensing of Land-Atmosphere Interactions; Choudhury, B.J., Kerr, Y.H., Njoku, E.G., Pampaloni, P., Eds.; VSP Press: Utrecht, The Netherlands, 1995; pp. 380-384.

25. Schiffer, R.; Thielheim, K.O. Light scattering by dielectric needles and disks. J. Appl. Phys. 1979, 50, 2476-2483.

26. Le Vine, D.M.; Meneghini, R.; Lang, R.H.; Seker, S.S. Scattering from arbitrarily oriented dielectric disks in the physical optics regime. J. Opt. Soc. Am. 1983, 73, 1255-1262.

27. Karam, M.A.; Fung, A.K.; Antar, Y.M.M. Electromagnetic wave scattering from some vegetation samples. IEEE Trans. Geosci. Remote Sens. 1988, 26, 799-808.

28. Karam, M.A.; Fung, A.K. Electromagnetic scattering from a layer of finite length, randomly oriented, dielectric, circular cylinders over a rough interface with application to vegetation. Int. J. Remote Sens. 1988, 9, 1109-1134.

29. Shi, J.C.; Jackson, T.; Tao, J.; Du, J.; Bindlish, R.; Lu, L.; Chen, K.S. Microwave vegetation indices for short vegetation covers from satellite passive microwave sensor AMSR-E. Remote Sens. Environ. 2008, 112, 4285-4300.

30. Zhao, T.J.; Zhang, L.X.; Shi, J.C.; Jiang, L.M. A physically based statistical methodology for surface soil moisture retrieval in the Tibet plateau using microwave vegetation indices. J. Geophys. Res. 2011, 116, D08116.

31. Li, X.; Zhang, L.; Weihermuller, L.; Jiang, L.M.; Vereecken, H. Measurement and simulation of topographic effects on passive microwave remote sensing over mountain areas: A case study from the Tibetan Plateau. IEEE Trans. Geosci. Remote Sens. 2014, 52, 1489-1501.

32. Zhao, T.; Zhang, L.; Jiang, L.; Chai, L.; Jin, R. A new soil freeze/thaw discriminant algorithm using AMSR-E passive microwave imagery. Hydrol. Process. 2011, 25, 1704-1716. 
33. Chai, L.N. Vegetation Biomass Inversion Algorithm Study Based on Passive Microwave Remote Sensing. Ph.D. Thesis, Beijing Normal University, Beijing, China, 2010.

34. Fung, A.K. Introduction: brightness temperature. In Microwave Scattering and Emission Models and Their Applications; Artech House: Dedham, MA, USA, 1994; pp. 16-18.

35. Ulaby, F.T.; Moore, R.K.; Fung, A.K. Microwave Remote Sensing-Active and Passive Volume I: Microwave Remote Sensing Fundamentals and Radiometry; Artech House: Dedham, MA, USA, $1981 ;$ p. 57.

36. Ferrazzoli, P.; Guerriero, L.; Paloscia, S.; Pampaloni, P.; Solimini, D. Modeling polarization properties of emission from soil covered with vegetation. IEEE Trans. Geosci. Remote Sens. 1992, 30, 157-165.

37. Ewe, H.T.; Chuah, H.T. Electromagnetic scattering from an electrically dense vegetation medium. IEEE Trans. Geosci. Remote Sens. 2000, 38, 2093-2105.

(C) 2015 by the authors; licensee MDPI, Basel, Switzerland. This article is an open access article distributed under the terms and conditions of the Creative Commons Attribution license (http://creativecommons.org/licenses/by/4.0/). 